\title{
The Meanings of the Gene and the Future of the Phenotype
}

\author{
LENNY MOSS ${ }^{1}$
}

\section{Introduction}

\section{Grasping at Complexity}

How does one analyze a living organism? It's not as easily settled a question as it may sometimes appear. For all the indisputable success of reductionist approaches in biology we are still not yet so very close to being able to explain how an embryo develops or even how a single cell functions. If biologists were able to build a living organism, even the simplest of living cells, out of purified parts, it would certainly do much to settle methodological and epistemological conundrums over questions of relative holism versus relative reductionism and presumably it would bring biology into a more seamless continuum with the physical sciences. Good intentions and boastful ambitions notwithstanding, we still cannot predict when that feat will be accomplished. And unless and until it is accomplished (and maybe even after that) the study of the living will be uniquely burdened with the dilemma of whether to try to grab onto basic units or parts, hypothetical or otherwise, and then proceed to work one's way 'up' to the complexity of a whole living system, or to begin at some minimum level of intact living complexity and attempt to poke it and probe it, hypothesize about it and take its measure in every conceivable fashion while yet preserving its integrity as a living system. Not that these need be mutually exclusive approaches - far from it. But how to relate the one to the other also remains an open question - bold claims by behavioural geneticists notwithstanding.

Earlier in the century, Niels Bohr famously speculated that there was no way to bridge these two standpoints, that rather, on the model of the wave and particle in quantum mechanics, they would be destined to co-exist independently, in a relation of perpetual complementarity. A protégé of Bohr, Max Delbrück, became a pioneer in structuring the physio-chemical investigation of life by way of an informationtheoretic outlook albeit with an on-going expectation that he would eventually hit up against the limits or 'paradox' that Bohr prophesized, ${ }^{2}$ which could well lead to the realization of new basic laws of physics. Inspired by Delbrück, Erwin Schrödinger ${ }^{3}$ proceeded to predelineate a new informational/linguistic vision of life with his anticipation of an 'aperiodic crystal' that would be the substrate of his so-called 'hereditary code-script'. For Schrödinger however, unlike Delbrück, the reduction of living complexity to bio-molecular information wasn't a strategy for recovering the truth of Bohr's complementarity at a more analytically refined level but rather was the entry-point into the promised land itself. ${ }^{4}$ Schrödinger's artful rhetoric did much to shape the terminological linguistic, and thereby conceptual, space in which the Watson and Crick breakthrough was received a decade later. Schrödinger's own point of departure, however, already presupposed a preformationistic understanding of the relationship of the genotype to the phenotype that he, somewhat naively, took to be the standing view of the genetics of his time. 
In addition to purely epistemological complexities (as if these weren't already hard enough), the understanding of the nature of the living organism is far too closely linked to questions of human self-understanding, an irreducibly normative affair, to be able to stand outside of the worlds of social and political interest. In other work I've suggested that the late nineteenth/early-twentieth century (re)turn to a preformationistic outlook in biology cannot be separated from the hardening and physicalization of social understanding expressed for example in the rising emphasis on race and racialized theories of history, along the contemporaneous formation of a eugenicist social agenda. ${ }^{5}$ I will return to the question of the significance of biological formulations for anthropological self-understanding at the end of the paper.

The concept of the gene ultimately has to be understood as arising in both epistemological and sociopolitical contexts, although I will only be able to focus on the former in this instant. A concept of the gene, no matter its particular formulation, is put forth as a possible handle with which to grasp hold of the complexity of the organism. Of course many issues turn on just how the nature of that grasp is construed. Both Weismann in Germany, and E. B. Wilson in the U.S., ${ }^{6}$ anticipated a gene-like concept prior to the turn of the century. There can be little doubt that by the end of the nineteenth century the time was ripe for something like a gene-concept to emerge. What Mendel's paper, rediscovered in 1900, provided was not merely another gene-type concept but a way to put a gene-type concept to work. Whether Mendel himself had conceived of his 'unit-characters' as the basis of a thoroughgoing analysis of the organism or merely as a practical device for aiding plant breeders, as Rafael Falk ${ }^{7}$ has argued, has been a subject of debate. Mendel's unitcharacter clearly opened a window onto something, but the nature and scope of that something was by no means defined or circumscribed by Mendel's findings. Mendel himself did not even distinguish between that which is transmitted in the seed and the traits, like red or white flowers, that eventually come to be seen. By simply referring to the transmission of unit-characters, as if it these were the traits themselves, eg, the red and white flowers, that were transmitted in the seed he spoke in the idiom of preformationism. But if indeed Mendel's intent was purely instrumental (as Falk as argued), there was no reason for him to do otherwise as it would merely have been a methodological shorthand.

\section{Johanssen's (non-preformationist) Gene}

The distinction between a 'genotype' and a 'phenotype'-between that which is physically transmitted in the germ and that which comes to appear with maturation in the next generation - was introduced by the Dutch botanist Wilhelm Johanssen who, in 1909, simultaneously introduced the term 'gene'. Johanssen distinguished between the genotype and the phenotype precisely to ward off the temptations of naïve preformationism, a fallacy that Johanssen never attempted to hide his disdain for. By distinguishing between a 'genotype' and a 'phenotype', Johanssen laid the groundwork for genetics to become a modern, independent and basic empirical science concerned with elucidating the relationship between that which is chemically transmitted from one generation to the next and the contextually conditioned realization of a particular organismic outcome from within a certain genotypically prescribed possibility space termed the 'norms of reaction'. ${ }^{8}$ Genotype, environment and phenotype thus always constituted a triadic relationship for Johanssen. Notably, it 
was this bit of conceptual housekeeping that quite likely induced T.H. Morgan, the embryologist and Mendelian skeptic, to 'convert' to genetics and become the leader of the new discipline. ${ }^{9}$ If Johanssen's phenotype/genotype distinction provided some conceptual leverage against encroachments of naïve preformationism, the exact nature and scope of the relationship of genotype to phenotype remained open and undecided. By 1923 it was clear to Johanssen, for example, that the phenotype cannot be fully decomposed down to an ensemble of separable units but rather that there was a core 'great central something' as he says, which could not and would not be decomposed. Let's take a look at what he had to say:

Certainly by far the most comprehensive and most decisive part of the whole genotype does not seem to be able to segregate in units; and as yet we are mostly operating with "characters", which are rather superficial in comparison with the fundamental Specific or Generic nature of the organism. This holds good even in those frequent cases where the characters in question may have the greatest importance for the welfare or economic value of the individuals.

We are very far from the ideal of enthusiastic Mendelians, viz. the possibility of dissolving genotypes into relatively small units, be they called genes, allelomorphs, factors, or something else. Personally I believe in a great central "something" as yet not divisible into separate factors. The pomace-flies in Morgan's splendid experiments continue to be pomace flies even if they lose all "good" genes necessary for a normal fly-life, or if they be possessed with all the "bad" genes, detrimental to the welfare of this little friend of the geneticist.

When we regard Mendelian "pairs", $\mathrm{Aa}, \mathrm{Bb}$ and so on, it is in most cases a normal reaction (character) that is the "allele" to an abnormal. Yellow ripe peas is normal, the green is an expression for imperfect ripeness as can easily be proven experimentally, e.g., by etherization... The rich material from the American Drosophiliaresearches of Morgan's school has supplied many cases of multiple allelisms - most of all of them being different "abnormalities" compared with the characters of the normal wild fly... To my mind the main question in regard to these units is this: Are experimentally demonstrated units anything more than expressions for local deviations from the original ("normal") constitutional state in the chromosomes?

Is the whole of Mendelism perhaps nothing but an establishment of very many chromosomal irregularities, disturbances or diseases of enormously practical and theoretical importance but without deeper value for an understanding of the "normal" constitution of natural biotypes? ${ }^{10}$

Now if Johanssen were right, it would not have thereby denied genetics the status of being scientifically or medically interesting and worthwhile but it should have set certain limits upon what Mendelian genetics was in the business of disclosing, that is, what it could be right about. If Johanssen were right, for example, then genetics 
would still be providing a certain kind of grasp-hold onto the complexity of the organism, but limited perhaps to certain relatively superficial aspects of it. If Johanssen were right then the truth of genetics would not be that of the wholesale genetic decomposability of the organism qua phenotype - and thus other ways of grasping onto that central core something would still be ultimately required.

One may want to surmise that Johanssen, writing thirty years before Watson and Crick, was expressing what is simply become an outdated view; but one would be mistaken. Rather, what will be argued is that precisely on the basis of the best contemporary understanding can we most fully appreciate just how prescient Johanssen's insights really were. Johanssen, of course, could not possibly have foreseen the breakthroughs that molecular biology has provided for grasping onto the inner-workings of the organism and yet there is one current meaning of 'the gene' consonant with what Johanssen articulated that continues to stand up to critical scrutiny. My interest in this matter is not particularly historical but rather pertains to how we now should best understand the relationship of genes to phenotypes. Invoking Johanssen's perspective is meant to serve as a kind of cognitive/perspectival resource. I will argue that on the basis of strictly current scientific and clinical usage that there are two distinctly different senses of the gene, that each discloses something about the phenotype, but that, properly understood, neither suggests that the phenotype can be decomposed down to an ensemble of genes - indeed quite the contrary. I will also suggest that much of the hyperbolic talk about gene as program, blueprints, and the like which has been very successful in finding its way to the public ear, is the result of an illicit conflation of these two meanings. I will then go on to consider some of the implications for our understanding of the phenotype that are derived from these gene concepts taken separately. Finally I will suggest that we are at the threshold of a new transformation in our understanding of the phenotype, and I will make some gestures in the direction of what the consequences this may hold for our theory of evolution and for our anthropological self-understanding.

\section{Gene-P versus Gene-D}

So what is this distinction? And what are these two gene concepts? When one speaks of a gene for blue eyes, or for cystic fibrosis, the gene for breast cancer, or for Marfan Syndrome, one is using a concept of a gene that is defined and specified by its relationship to a phenotype. I call this sense of the gene, 'Gene-P'. What allows something to satisfy the conditions of being a Gene-P is some predictable relationship to the appearance of a certain phenotype. While defined by a predictable relationship to a phenotype, Gene-P, by contrast, is indeterminate with respect to DNA structure, that is, with regard to specific nucleic acid sequence. The $\mathrm{P}$ in Gene-P stands for 'preformationist'. To speak as if the transmission of a gene were tantamount to the transmission of a phenotypic trait is to speak in the idiom of preformationism. Preformationism, a child of the seventeenth century, taken to its extreme would hold that all of the traits of the mature organism are already determined and present at birth. The proper contemporary use of Gene-P, however, is not an expression of the ontology of preformationism but only of an instrumental deployment of it. To speak of a Gene-P for a phenotype is to speak as if, but only as if, there were a definite certain something that was transmitted between generations and that dictated a 
distinct phenotypic outcome. But as we've already said, Gene-P is indeterminate with respect to its physical referent. It is indeterminate with respect to its DNA sequences and so there is not a certain something that is being transmitted, but rather an uncertain something. How can this be? Would it not simply be a matter of empirically fleshing out what the structure of the physical referent would be? The answer is no. And the reason the answer is no is that what one almost always, if not always, finds in cases of Gene-P, is that it is not the presence of some specific sequence that correlates with the appearance of a phenotype, but rather the absence of some 'normal' sequence resource that is at issue, and there are always many ways for something to be absent. There are, for example, over 900 different documented DNA sequences that may show up as 'the gene for cystic fibrosis'. Likewise, the assurance that two blue-eyed parents may be given that a child of theirs would also have light-colored eyes is based upon the assumption that neither blue-eyed parent has the genetic resource for producing brown-eye pigment, however it is that they may be lacking it. What is inherited, what is passed on, is the lack of something, the lack of a normal resource for producing brown eye pigment. To heritably lack the wherewithal for producing brown-eyes is thereby to possess the Gene-P for blue eyes. As Johanssen suggested, Genes-P can be of great medical and or economic significance but they are essentially, allelic abnormalities that do not provide the basis for decomposing the core of an organism.

The genes of classical genetics were Genes-P, but Gene-P is no longer limited to classical methods. The disciplinary context in which the meaning of the gene as Gene$\mathrm{P}$ is the most clear-cut is the medical genetics clinic. In the medical genetics clinic, Gene-P is used as a predictor of phenotypic outcomes such as the likelihood of having a child with cystic fibrosis or of contracting breast cancer given a family history. In the modern genetics clinic, traditional family pedigrees can be supplemented or even replaced by the use of molecular probes. Molecular probes are targeted to a specific sequence, but because a Gene-P is indeterminate with respect to sequence, because a Gene-P is characteristically based on the absence of some sequence, probes can only probe for particular ways of not having the normal sequence and never fully rule out the possibility of some other way of not having the 'normal' sequence. What genetic probes can do is to survey some set of the relevant ways that a "normal" sequence may be absent. If there are 900 documented ways for the normal sequence to be absent resulting in a Gene-P for cystic fibrosis, then it would require 900 different probes to exhaust the possibility of there being a Gene-P for cystic fibrosis given current knowledge - although in practice this is modified by statistical data pertaining to the frequency of particular mutations in different populations.

Whereas Gene-P is associated with, indeed defined by, a phenotype and thus brings the notion of the 'phenotype' into the semantic reference space of the word gene, Gene-D, by contrast, is defined by molecular, ie, DNA, sequence but is indeterminate with respect to phenotype. If some stretch of DNA provides the template out of which a strand of RNA of complementary sequence is produced, then for a biologist, working at the cell and molecular level, this counts as a gene. To count as a Gene-D entails this and nothing about the ability to predict a phenotypic outcome. But why would that be? And again, might it not be the case that the lack of an ability to specify a phenotypic trait on the basis of DNA sequence is just due to a lack of knowledge? 
A possible, in-principle explanation for why a DNA sequence does not determine a specific phenotypic trait would be that any particular DNA sequence can in itself only contribute to any number of different, and often even antithetical, phenotypic outcomes and thus can never be adequate to the task of narrowing down, or specifying, which of these would come to be the case. The ' $\mathrm{D}$ ' in Gene-D stands for 'developmental resource' and is meant to evoke exactly this type of reasoning. Whereas Gene-P does its conceptual work through offering a peformationist standpoint, but only instrumentally and predictively, as the best we can do in the absence of a full-fledged molecular developmental account of an often pathophysiological outcome, Gene-D assumes a causal-explanatory framework which seeks to elucidate exactly that full-fledged molecular-developmental account, but in order to do so must abstain from delegating causal privileges or priorities prior to the achievement of the empirical account itself. Study upon study at the cell and molecular level shows that any particular Gene-D can participate in, and contribute to, a myriad of different, and even antithetical, phenotypic outcomes and cannot in itself determine which phenotypic outcome it will ultimately contribute to.

The character of Gene-D, and why it is indeterminate with respect to phenotypic consequences, is best illustrated with the help of an example. My example called NCAM (for the neural cell adhesion molecule) is representative of a very large class of Genes-D called 'cell adhesion molecules' of which more will be said when the lessons or implications of Gene-D are discussed. NCAM is a member of a very large family of homologous genes called the Immunoglobulin Superfamily (IgSF). ${ }^{11}$ The IgSF is one of three such families of related genes associated with cell-adhesion functions, the other two being called the 'cadherins' and the 'integrins'. Humans, and other vertebrates, have only one Gene-D for NCAM in their genome (although as diploid organisms we would have two copies of it). But there are multiple ways in which the very same genetic template can be put to very different uses, which is the point of this example. Like the great majority of molecular genes, that is, Genes-D, NCAM is composed of multiple exons which are the modular units that actually contain the template information for synthesizing messenger RNA and thereby protein. The NCAM gene consists of nineteen exon units. ${ }^{12}$ In the process of synthesizing an NCAM protein, first an mRNA transcript is produced in the nucleus that contains all 19 of the NCAM exons. But there are no NCAM proteins that contain the products of all 19 exons. The contingent functional specification of the product of a gene begins with the splicing out of certain exons from the RNA transcript - a process that is not and cannot be determined by the gene itself. There are many different ways in which cellular context makes all the difference with respect to what the effects of NCAM gene expression are going to be in any situation. The first of these, we've already seen, pertain to which isoform is expressed, which depends upon the complex of proteins that are involved in splicing. Hundreds of different isoforms of NCAM in mammals have been identified. ${ }^{13}$ Characterizing the scope of variability of isoforms derived from differential splicing is still in its infancy and will ultimately have much to say about the relationship of genomes to proteomes. As a taste of things to come, the cell-adhesion molecule called DSCAM found in Drosophila, the famous fruit fly, is capable of giving rise to 38,000 different isoforms, a great many of which have been identified at the messenger level. ${ }^{14}$ These isoforms are found on the surface 
of embryonic retinal cells as they extend outward in the exploratory formation of synaptic connections in the development of the compound eye of the fly. The fact that a single Gene-D can give rise to a greater number of isoforms of a protein than there are genes in the human genome should be quite suggestive as to where post-genomic biology will be heading. But how and why does variation in isoform matter? The different domains of NCAM that are coded for by one or two exons are associated with different biochemical and physiological activities. The NCAM protein is associated with the cell membrane, with most of its length extending outside of the cell where it may come into contact with other cells. How the NCAM is spliced will affect how, and whether, the protein is attached to the cell membrane and also how much of the protein will extend into the interior, ie, the cytoplasmic compartment of the cell. As each of these domains carries its own functional portfolio, variation in the domains expresssed will have direct and indirect ramifications on the phenotype. Of central importance in the developmental physiology of neural tissue is the balance between neural plasticity, ie, the ability to form new synapses versus neural synaptic stabilization. ${ }^{15}$ Without the ability to form new synapses, neural development simply could not take place in the embryo but neither could new learning take place in the adult. However, if there is no synaptic stabilization, then stable behavior patterns could not become established, skills and habits could not be learned and acquired, and memories could not be retained. Now even though all of the NCAM proteins come from the same Gene-D, the form they take will make all the difference as to whether they are contributing to the stabilization of synapses, or to synaptic plasticity, and in either case as to what kinds of synapses are being formed.

The largest of the NCAM isoforms, those that are designated as ' $180 \mathrm{KDa}$ ' in size, contain the domain coded for by exon number eighteen and contribute to synaptic stabilization as that domain interacts with cytoskeletal components within the neuronal cell. Far more dramatic, however, is the consequence of whether or not the product of the fifth exon domain becomes decorated with large negatively charged chains of polysialic acid (PSA). NCAM isoforms lacking the eighteenth exon domain but heavily decorated with polysialic acid predominate through embryonic development. In the brain of the adult rat, polysialylation is found only in regions that continue to undergo structural rearrangement, ie, in regions associated with new learning. For the rat, whose world is principally disclosed through the olfactory sense, unlike that of the visually-dominant human, the olfactory bulb is a hotspot for structural rearrangement.

The loss of PSA-NCAM (polysialylated NCAM) - a change that is independent of any changes in the Gene-D for NCAM itself-is associated with the loss of neural plasticity. ${ }^{16}$ What is true for NCAM is true for Genes-D in general and for other cell adhesion molecule genes in particular. Cell and developmental context will influence not only what structural form of a cell adhesion molecule is realized from a multivalent genetic template but even how any particular isoform will function. In the 2003 volume of the review journal Current Opinion in Cell Biology, in the issue dedicated to the biology of cell adhesion, the Editors summarily observed that 'In general, the cellular context can greatly influence the adhesive and signaling properties of a given molecule. The same cadherin behaves differently in different cell types; and the same is true for integrins and other adhesive proteins'. 


\section{What Must be True about Organisms for Genes-P to Exist?}

If a gene, empirically understood as a piece of cellular chemistry that has the capacity to serve as a template for RNA synthesis, ie, as a Gene-D, does not and cannot determine a phenotypic trait in itself, then how, one may well wonder, can it even be possible for a Gene-P to exist? Is Gene-P a special subset of Gene-D? If not, then what is it that causes the phenotypic outcome that defines Gene-P? What has to be the case about an organism for Genes-P to exist? And what is the relationship between Gene-P and Gene-D?

Throughout most of the twentieth century elucidating the genotype had been the central strategy for understanding the phenotype. Following Mendel's lead, classical genetics parsed the phenotype into traits precisely according to whether such parsing was serviceable for further elucidating the genotype. Parsing the phenotype according to the rules of Mendelian inheritance gave birth to Gene-P. There was nothing in the experience of classical genetics that warranted the assumption that a phenotype would be fully decomposable into genotypic units and Wilhelm Johanssen, as we've seen, was dead certain that it would not. However, so long as classical genetics could see an open road ahead for further elaboration of the genotype qua Gene-P, and so long as no more powerful approach for understanding the phenotype came on the scene, nothing stood in the way of continued attempts to decompose the organism into so many Genes-P.

This enterprise took a new turn with the characterization of DNA as the heritable source of templates for synthesizing protein, and thus the arrival of Gene-D. Despite the de facto independence of Gene-D as an organizing concept in molecular-level biology, the continued primacy, during the last half of the twentieth century, of the genotype as the means for understanding the phenotype had been based largely on the de facto conflation of Gene-D with Gene-P. While the idea that the phenotype could be fully decomposed into a conglomeration of genes, each of which roughly codes for a trait, began with those zealous Mendelians that Johanssen took to task, in the second half of the twentieth century it has been the conflation of Gene-P with Gene-D that has given rise to the idea of the master-molecule, or blueprint, that is specified simultaneously by its nucleic-acid sequence and its phenotypic consequence. It is this conflationary view of the gene, which borrows and blends from two categorically different disciplinary contexts within biology and medicine, that underlies the genecentered conceptions that have been advanced by certain evolutionary biologists and philosophers, certain evolutionary psychologists, and which has been given a broad public exposure by the likes of popularizers such as Richard Dawkins, Daniel Dennett and Steven Pinker as well as large numbers of science journalists.

Looking ahead into twenty-first century post-genomic biology, beyond the conflation of Gene-P and Gene-D, the phenotype itself has become poised to take center stage. The sequencing of the human genome and the move to genomics can well be seen as the culmination of an enterprise and the exhaustion of a research strategy. For many 'Systems Biology' has become the research programme, heir apparent. Ironically, perhaps, it is precisely the empirical knowledge gained within the disciplinary context 
of Gene-D that allows us to definitively disambiguate and de-conflate Gene-P and Gene-D. Where for a century much of biology has been structured around the attempt to understand the phenotype on the basis of the genotype, with the culmination of this enterprise in genomics and with the disambiguation of Gene-P and Gene-D a new horizon presents itself. Gene-P and Gene-D, considered separately, each opens a window, albeit very different windows, onto the complexity of the phenotype. The analysis of the genotype can longer be taken as a substitute for that theory of the phenotype that we have yet to realize. Unburdened with the shackles of conflation, both Gene-P and Gene-D can provide new points of entry into the construction of a theory of the phenotype. Beyond conflation, we can now ask what is illuminating about Gene-P and about Gene-D?

\section{Listening to Gene-P}

\section{The Case of Marfan Syndrome}

Examining two very different examples of Genes-P will help to address the questions that I've posed about the nature of Gene-P, its relationship to Gene-D, and what kind of window onto the phenotype each gene concept provides when no longer conflated with the other. The two Genes-P that I will discuss are the genes for Marfan Syndrome and BRCA1, the gene for breast cancer. Marfan Syndrome is also known as Abraham Lincoln disease. It was first characterized by Antoine Marfane in 1896 in a five-year-old girl possessed of unusually long tapering fingers. Marfan syndrome ${ }^{17}$ appears in one in ten thousand individuals worldwide. It displays a cosmopolitian range of distribution, showing no preference with respect to region, nationality, or gender. Marfan syndrome is characterized by a set of traits, the expression of which are highly variable (pleiotropic). Most characteristic of Marfan Syndrome is a tall, thin stature, sometimes athletic. The face tends to be narrow and the palate high. While it isn't known whether Abraham Lincoln was in fact a Marfan, it is from these features that the association with Abraham Lincoln is derived. Like the five-year-old French girl mentioned above, Marfan individuals will frequently have unusually long limbs with long and tapering fingers and toes. Other features include a breastbone that is either pushed in or pushed out, a propensity for scoliosis, joints that are unusually loose and somewhat injury prone, off-center lenses in the eye and myopic vision and - the medically most significant trait - is susceptibility to aortic aneurism that can only be detected by an echocardiogram. ${ }^{18}$

Marfan Syndrome is a disease of connective tissue. It is the result of a failure to properly incorporate the connective tissue protein fibrillin-1 into the microfibrils of developing connective tissue throughout the body. There are at least 150 mutations of the Fibrillin-1 gene (Gene-D) associated with Marfan Syndrome. ${ }^{19}$ Any of these could 'show-up' as a Gene-P for Marfan Syndrome. As is typical of a Gene-P, any of these genes for Marfan Syndrome can be tracked by pedigree analysis or by molecular analysis given the right molecular probes. What makes a gene a 'gene for MarfanSyndrome,' that is, a gene for a certain phenotype and thus a Gene-P, is its predictive relationship to a phenotype. But let us consider just what is the relationship of a gene for Marfan-Syndrome to the phenotype. What is it that causes the characteristics associated with the phenotype? Is there any good sense in which a gene for Marfan Syndrome can be construed as carrying information or coding for a trait? Does the 
gene for Marfan Syndrome tell the developing body to be tall and lanky, or to have a high palate or to be near sighted? Certainly not. The features of Marfan Syndrome, like that of blue eyes, are the results of what human bodies do in the absence of a certain otherwise typical molecular resource. The wherewithal for responding to this absence is inherent in the complex systematicity of the phenotype, in its adaptive plasticity, properties that simply cannot be decomposed down to atomic units. The Gene-P for Marfan Syndrome reveals this systematicity, this capacity, given a certain constraint or perturbation, for the developing phenotype to developmentally 'style itself' in a recognizable way. There is no gene that codes for an Abraham Lincoln phenotype, but Marfan Syndrome reveals that the systematic properties of the human phenotype are such that, given a certain class of perturbations, some set of Marfan characteristic will result. Marfan Syndrome is an expression of the polyphenism of the human phenotype, that is, its ability to phenotypically shift directions while yet retaining its overall integration as a functional system. Within a context of 'family resemblance', Marfan individuals will vary with respect to specifics. Individuals who differ with respect to which of the 150 mutations they carry are seen to show phenotypic differences, yet some individuals with the same mutation will also differ and some individuals with different mutations will present a great deal of resemblance. In addition, a Marfan phenotype may be found in individuals without a mutant fibrillin-1 gene at all, because any perturbation, genetic or otherwise, that interferes with fibrillin synthesis, transport, or microfibril assembly can lead to the same type of polyphenic outcome.

Now what about the Gene-D for fibrillin? If the example of Marfan Syndrome has made good on the claim that a Gene-P is indeterminate with respect to molecular structure or sequence - and that it is only an instrumental predictor of the phentotype it is associated with - what does the Marfan example have to say about the claim that any Gene-D will be indeterminate with respect to phenotype? As is the case in relation to any, or almost any, Gene-P, there is no corresponding normal gene with specificity for a phenotypic contrast class. The Gene-D for fibrillin-1, ie, the normal gene, does not, for example, favor a short and squat phenotype any more than it favors a tall and slender one. The Gene-D for the fibrillin-1 template sequence can be used in many different ways in different contexts and it will always be the larger developmental context that will determine toward which phenotypic outcome the gene-product will contribute.

\section{The Case of BRCA1}

BRCA1, the 'gene for breast cancer', provides another useful example because it differs from the gene for Marfan Syndrome in all its particulars and yet the take-home lessons about the relationship between Gene-P and Gene-D, and what it is that a Gene-P reveals about the phenotype, are quite consistent. BRCA1 has been the flagship gene for the enthusiasts of germ-line genetic testing. ${ }^{20}$ A positive result with a molecular probe test for the presence of a BRCA1 gene correlates with an $85 \%$ likelihood that a woman from a family with a history of breast and/or ovarian cancer will contract breast cancer at some point during her own lifetime. Defined by this phenotypic prediction, BRCA1 or the gene for breast cancer is a Gene-P. As is the case with other Genes-P, there are numerous mutations (at the BRCA1 locus) that meet the criteria for being a gene for breast cancer. Owing to the interest that BRCA1 
has garnered since first being identified, the molecular biology of the Gene-D, which alas is also called BRCA1, has been intense since it was first identified over 20 years ago. Unlike fibrillin, which is secreted into intercellular space and becomes a structural component of the body's connective tissue, BRCA1 (the protein) is active in the nucleus of the cell. The distribution of BRCA1 with respect to tissue type and developmental stage is described as 'ubiquitous'. Just as the Gene-D for fibrillin is not a gene for short and stocky phenotypes, but like any Gene-D is indeterminate with respect to phenotype, the "normal" BRCA1 is not a gene for healthy breasts, nor does it show any specificity for breasts at all. BRCA1 is a large multi-modular protein that has been implicated in several different functions within the nucleus - transcriptional regulation, DNA repair, chromosome remodeling, and the targeting of proteins for intracellular degradation - and it is apt to be found in every cell in every tissue in the body. ${ }^{21}$ The tips of the BRCA1 protein have the capacity to bind to the RNA polymerase enzyme, and the middle region of the protein has been shown to bind to a wide variety of both transcriptional enhancers and transcriptional repressors.

The interesting question, then, becomes: why do mutant germline forms of BRCA1, while present and active in every type of cell in the body, show up as a Gene-P specifically for breast cancer? ${ }^{22}$ What does this Gene-P disclose about the nature of the phenotype (and perhaps about cancer as a potential inherent within the phenotypic possibility space)? The BRCA1 protein in the nucleus of the cell has been shown to serve as a kind of platform upon which other proteins assemble in stage- and tissuecontingent ways. The number of proteins that interact with BRCA1 has been described as astronomical. What becomes suggestive is the possibility that what may best account for why germline mutation in the BRCA1 gene shows up as a Gene-P exclusively for breast cancer can be best approached in terms of the role of the BRCA1 protein in a complex network of interactants. In other words, is the Gene-P for breast cancer giving us a porthole, not onto the decomposability of the phenotype, but onto previously invisible, network-theoretic properties of the phenotype?

We will borrow from the scale-free network analysis developed by the physicist Albert-László Barabási. ${ }^{23}$ In studies of the proteome, that is the entire ensemble of expressed proteins, of the single-celled organism yeast, Barabási and co-workers ${ }^{24}$ looked at yeast proteins simply in terms of the number of the other proteins that any particular protein interacted with. It has been typical of Barabási's studies of networks of all kinds, from social networks, airline flight patterns and the Internet, to living cells, to find that most nodal points, usually around $80 \%$, have only small numbers of linkages to other nodes, whereas the remaining $20 \%$ are linked to many other nodes and thus constitute the 'hubs'. The 'diameter of a system', network-wise, can be reckoned in terms of the maximum number of links that it would take to connect any two nodes in the system. Hubs would clearly play a critical role in maintaining a fixed diameter of a system and when, for example, yeast species have evolved the ability to metabolize new substrates, investigators have found that systematic adjustments take place in the metabolic network such as to enable the diameter of the network to remain constant. ${ }^{25}$ In further examining yeast, Barabási and coworkers found that $93 \%$ of proteins have five or fewer links, whereas only $7 \%$ of proteins have 15 or more links. Not surprisingly, the deletion of only a small percentage of the weakly 
linked proteins proved to be lethal to the yeast, whereas the deletion of the majority of the hub proteins proved to be lethal.

Although purely speculative at this point, suddenly we have a completely new model for what a Gene-P might be revealing about the phenotype. First of all, for something to show up as a Gene-P, it cannot be a developmental-lethal. If a gene product served as a 'hub' in every tissue, or in any developmentally vital process, its absence would likely be lethal and so not show up as a Gene-P at all. Breast cancers associated with germ line mutations of BRCA1 are quite variable in terms of age of onset, but seldom if ever, occur before puberty and most often occur beyond a woman's childbearing age. It could turn out to be the case that BRCA1 isoforms play a sensitive role in maintaining something like a uniform network diameter in the nuclear regulatoryprotein system of post-pubescent mammary cells. Because the post-pubescent mammary gland will constitute its own distinctive biochemical context, it is wholly plausible that an otherwise ubiquitous molecule could play a distinctive role from a network perspective. Should this prove to be the case, then it would simply be a kind of quirk about the systematic relationships of regulatory proteins in the nucleus of mammary cells that would result in BRCA1 mutations showing up as Genes-P for breast cancer, but it would be a quirk that could allow this Gene-P to reveal something hitherto invisible and unimagined, not about the decomposability of the phenotype, but, quite to the contrary, about the systematic, and thus indivisible, properties of the phenotype resident in every nucleus in every cell of the living body.

\section{Interrogating Gene-D}

Finally, what window or windows onto the phenotype might be revealed by Gene-D when no longer burdened by conflation with Gene-P? In a recent article entitle 'Genes Classical and Genes Developmental: The Different Use of Genes in Evolutionary Syntheses', the developmental biologist, textbook author, and historian of biology Scott Gilbert, ${ }^{26}$ writing in a largely pluralistic mood, provides a perspicuous comparison between the beliefs about genes held by the population geneticists who advanced the 'Evolutionary Modern Synthesis' and those of the contemporary molecular biologists who are trying to advance a new evolutionary-developmental, or Evo-Devo, synthesis. Gilbert distinguishes these as 'Population Genes' versus 'Developmental Genes'. I will just pick out some of the more salient features of his comparison. The population genes, whose physical referent were hypothetical, were thought to be atomistic units responsible for distinctively different enzymes and structural proteins that would result in distinctively different phenotypic traits with different fitness values for adult organisms competing for survival. By contrast, the developmental genes, being characterized by molecular biologists interested in evolution have been found to show great similarity, not difference, across taxa; to be associated not with enzymes and structural proteins but with signaling and the regulation of transcription and splicing; to be most significant in their expression not in the adult but in the embryo; and to be context-dependent parts of a pathway rather than acting in a context independent atomistic fashion, as the population geneticists had assumed. 
It should be evident at this point that what Gilbert has described as the presuppositions of population genetics about the nature of the gene are exactly what I've described as coming to constitute the conflationary view. What Gilbert has described as the developmental gene is exactly what has emerged out of the disciplinary context of Gene-D. ${ }^{27}$ But in seeking to clarify the further implications of Gene-D for understanding the phenotype, we can supplement the characterization that Gilbert has provided with a comparative analysis of the findings of different genome projects and other studies that have made evolutionary comparisons from the point of view of Gene-D. After all, the output of genome projects is simply compendia of Genes-D, and one way of asking what Gene-D can tell us about the nature of the phenotype is to see how these compendia change over evolutionary time. ${ }^{28}$

\section{Comparative Genomics and Gene-D}

When we look at organisms of different levels of complexity, single-celled to multicellular, invertebrate to vertebrate, fish to mammal, mouse to human, what kinds of changes in the genomes appear to coincide with changes in the complexity of the organism? The Human Genome Project upset traditional expectations by revealing that humans have only twice as many genes as the fruit fly and only one third more than the tiny nematode worm, C. elegans. ${ }^{29}$ Genes-D, as we've already mentioned, are composed of modular units called exons, and it turns out that even this very moderate difference in gene number is based largely on the reshuffling of old exons to produce new combinations, with less than $7 \%$ of the increase in gene number based on apparently new modules. So, what we see at the level of genomic differences is that what appears to correlate with changes in complexity is not gross increases in novel genetic resources but rather the intensification of the ability to use the same basic resources in a greater number of ways. What we see at the level of comparative genomics becomes greatly magnified at the level of comparative proteomics. While vertebrates, including humans, have only at most twice as many Genes-D as invertebrates, the complete set of expressed proteins derived from the vertebrate genome appears to be at least five times as great as that derived from the invertebrate genome. The difference between genome size and proteome size would be largely accounted for on the basis of the ability to generate multiple isoforms through differential RNA splicing, as we previously discussed. So, we now know that phenotypes of different levels of complexity are composed of largely the same set of modular resources, but are used in increasingly flexible and nuanced ways. Can the window that Gene-D opens up on the phenotype give us any more insight into how this expansion of the use of genetic modularity is distributed? Is it, for example, evenly distributed across the genome or is it lumpy? And if it is lumpy, does the lumpiness have a meaning that we can interpret? By the looks of some recent studies, the answer to these questions appears to be yes.

A systematic comparison ${ }^{30}$ between the DNA sequences of human chromosome 19 and those of the homologous chromosomal regions of the mouse suggests that the ensemble of Genes-D that constitute a genome fall into two distinct categories. On the one hand, there are unique, single copy-genes that are dispersed through the genome. On the other hand, there are families of closely related genes that are grouped together on the chromosome in tandem clusters. In comparing the human and mouse 
sequences, what was found was an "overwhelming" conservation of sequence of single-copy genes and extensive differences in genes residing in tandem familial clusters. Given what we have already observed, a little bit of extrapolation would not be out of order. What appears to be the case is that a core set of single-copy genes has been a constant throughout the animal kingdom going back to the most primitive stages. Might it be the case that exactly those genes that provide the templates for basic enzymatic and structural capacities that the population-theoretic architects of the Evolutionary Modern Synthesis assumed would be the basis of evolution, are precisely those that have by and large not evolved at all?

The evolutionary history of Genes-D appears to be associated with the expansion and contraction of differentiation of families of closely related genes or 'gene-families' that are found to be present in tandem clusters on chromosomes. Is there any specificity to which gene-families are found to expand in relation to apparent evolutionary increases in organismic complexity? In their 2002 report in Science magazine, J.A. Bailey et al. found that the expansion of gene families comes about through an incomplete duplication of genes, referred to as segmental duplication, that results in the reshuffling of modular exon units and thereby the production of greater protein diversity. ${ }^{31}$ They found that such segmental duplication is not distributed randomly throughout the genome. Rather they report that 'genes associated with immunity and defense, membrane surface interactions, drug detoxification, and growth/development were particularly enriched'. While there are doubtlessly interesting things to say about all four of these categories, I will focus briefly on one of which we've already introduced.

Cell adhesion molecules constitute an important subset of those gene families associated with cell surface interactions. The Immunoglobulin Superfamily (IgSF), of which I said NCAM is a member, would come under the heading of two of the groups cited above - membrane surface interactants and genes associated with immunity and defense. ${ }^{32}$ Let us consider what one can construe about the nature of the phenotype through the porthole that the Genes-D of the IgSF provides. Whereas humans have only double or fewer the number of genes overall than the fruit fly or worm, there are 765 IgSF genes in the human genome compared with 140 for fly and 64 for worm. It is estimated that the number of isoforms of proteins from the human IgSF alone would exceed the entire genome size of both fruit fly and nematode worm. All indications are that the comparative findings for other cell adhesion gene families, the cadherins and the integrins, would be quite similar. But what might this tell us about the nature of the evolving phenotype? To answer this question, imagine first that a cell (cell \#1) has a single receptor on its surface that is capable of binding to some closely related range of ligands (a ligand simply being a molecule with which a receptor may bind). And let us say that when the receptor binds to the ligand, it can transmit a signal into the cell that results in some change in the physiological and/or transcriptional state of the cell. Now, let us compare this to two other scenarios. In the second scenario (cell \#2), let us imagine that for the same range of ligands that our one receptor could bind to, we now have 10 different receptors, each of which specializes in binding some subset of that same range of ligands, and that each of these receptors can then transmit a different signal, thus one of ten different signals, to the interior of the cell. For our third scenario (cell \#3), let us imagine that we now 
have ten different receptors that vary, not with respect to their binding specificity, but only with respect to the signal that they transmit back to the interior of the cell.

Taken together, scenarios two and three (cells \#2 \& \#3) represent a marked increase both in the sensitivity of the cell to nuanced differences in its surround, properties that could also be described as an increased 'openness' of the organism to its environment or its world, and in a measure of increased autonomization, whereby the internal state of the cell can determine which receptors to use to probe its surround, a property that could also be described as increased 'detachment'. ${ }^{33}$ I've been speaking of the cell as if it were a kind of microcosm of the whole organism in its relationship to an environmental surround, but let's also think of it as the unit of multicellular development. A developing organism's developmental trajectory is largely realized through the interaction of its cells with each other and with aspects of their environmental surround, ie, their 'world.' Where an organism is composed of cells that have evolved the capacities of cells \#2 \& \#3 (as opposed to merely that of cell \#1) there would be an enhanced capacity for making fine distinctions about their surround, for executing internal-state-dependent 'choices' about how to respond to even the same stimuli, and thus a massively greater capacity for phenotypic flexibility, responsiveness, and adaptability. The evolutionary expansion of the repertoire of cell-surface adhesion and signaling receptors with the IgSF and other gene-superfamilies can be seen as a key factor in the evolution of 'world-openness and 'detachment' ie, as what we experience as increasingly complex forms of life.

\section{Phenotype First?}

The analysis of Gene-P and Gene-D aspires to provide a perspective with which to break bread once again with Johanssen and rethink the meaning of genes and phenotypes from their roots up. I argued that from at least two different angles we can see why the question of the phenotype is back on the table. What Gene-P and Gene-D both reveal, albeit in different ways, is that there are systematic, self-organizing capabilities of the phenotype which will not yield to the kind of decompositional approach that has held sway during much of the past century, nor for that matter any gene-centered approach at all. Without even attempting to survey exhaustively the relevant sources, I will mention a few of the more salient examples.

In the 'Millennium Issue' of the prestigious molecular biology journal Cell, Marc Kirshner and Tim Mitchison of Harvard and John Gerhart of Berkeley, ${ }^{34}$ criticize the limits of the machine metaphor in biology, and describe their postgenomic proposal for an integrated cell and organismal physiology, somewhat light-heartedly, as 'molecular vitalism'. What is not light-hearted for them is the idea that the phenotype needs to be understood on its own terms, and structured neither on the basis of the machine metaphor nor that of genetic information. In their more recent The Plausibility of Life, Kirschner and Gerhart ${ }^{35}$ draw on the latest findings in cell and developmental biology to explain the active role of organisms (ie, of phenotypes) in 'facilitating' the production of evolutionary variation.

In their anthology Origination of Organismal Form. Beyond the Gene in Developmental and Evolutionary Biology, theoretical biologists Gerd Müller and 
Stuart Newman, ${ }^{36}$ along with contributors to their volume, have been exploring the idea that organismal form originates with the inherent properties of living tissues, ie, of the phenotype, and that genes are secondary factors whose role began largely as that of stabilization. Likewise, contributors to the recent anthology called Cycles of Contingency ${ }^{37}$ share the inclination to recontextualize genes, understood in a Gene-D kind of way, as one resource among many, in conceptualizing the dynamics of developmental systems. The most sustained and impressive effort of all, however, has been that undertaken by biologist Mary Jane West-Eberhard, ${ }^{38}$ whose 800 page tome is entitled Developmental Plasticity and Evolution. West-Eberhard's goal is nothing less than a radical transformation of our theory of evolution based upon a phenotypecentered biology.

Much of West-Eberhard's painstaking study is devoted to documenting the ubiquity of polyphenisms across all living taxa. A polyphenism is the ability of an organism to undergo radical shifts in its phenotypic expression. This often involves shifts in developmental timing that can have drastic effects on the phenotype including the entire loss of a later stage of development. Of the 113 species of salamander, for example, that undergo metamorphosis, $37 \%$ of these species are found to have adults that do not always undergo metamorphosis. The loss of a later stage of development is known as neoteny, and it has played a major role in various evolutionary events and transitions (including human evolution as discussed below). Polyphenism may be a source of both de novo evolutionary innovation or the redeployment of phenotypic forms already present within a latent developmental and/or ecological phenotyperesponse repertoire. There are, for example, 27 genera of fish with facultative airbreathing capabilities. The colonization of land was achieved in her view, first of all, not through the evolution of a new species, but rather by way of polyphenic adaptations by a species already invested with facultative air-breathing capability. The capacity for breathing on land was already 'anticipated' and multiple times, by genera of fish most of whom did not give rise to land inhabiting species.

The systematic capability of the phenotype to undergo adaptively plastic transformations in response to a new input, in West-Eberhard's model, constitutes the principal source of evolutionary innovation, thus shifting much of the adaptive and innovative centre of gravity back to the systematic capacities of the organism itself. She suggests a four-stage model of adaptive evolution in which genes may play two decidedly different roles. These roles, as it happens, correspond well to my Gene$\mathrm{P} /$ Gene-D distinction. These stages are as follows:

1. New Input:The phenotype is exposed to some form of perturbation that could be either environmental or genetic. If genetic it would be in the sense of Gene-P.

2. Phenotypic Accommodation: The organism draws upon its plastic, polyphenic capability to respond adaptively to the challenge.

3. Recurrence or Initial Spread: The phenotypic shift spreads through the population either due to the recurrence of the initial input or the spread or other means of inducing the phenotypic shift. To this I would add the suggestion that the phenotype-illiciting input could become stabilized as part of the organisms 'constructed niche', which in the case of humans means cultural institutionalization. 
4. Genetic Accommodation: Unlike the initial input, which could be a single mutation or an environmental cue, that triggers the phenotypic shift, genetic accommodation takes places at multiple loci (this would now be the Gene-D sense of genetic). Genetic Accommodation occurs simply by way of natural selection (i.e., differential survival) occurring within the context of the new selective regime introduced by the phenotype. The genetic heterogeneity is that which was already present in the population prior to the phenotypic shift. Genetic accommodation improves the novel phenotype in three or more different ways. These are: a) adjusting regulation, to change the frequency of expression of the trait or conditions under which it is expressed, b) adjusting the form of the trait, its integration and efficiency, c) reducing disadvantageous side effects.

The resonance between West-Eberhard's model and my distinction between, and analysis of, Gene-P and Gene-D, shouldn't be too difficult to discern. West-Eberhard thinks that the most likely source of the initial input is environmental, and she may well be right, but she considers a genetic mutation to be a relevant candidate as well. This depiction of a mutation constituting, not new information for the phenotype, but a perturbation to which the phenotype responds in a coherent systematic fashion, is exactly in tune with the characterization of Gene-P, as is the symmetry she depicts between such a mutation and an environmental shift (ie, two types of 'perturbations') that could trigger the same response. The process of genetic accommodation, as she understands it, entails selection at multiple loci, which is also to say that none of the loci, independently has a determinate relationship to the resulting phenotype. Genetic accommodation, sensu West-Eberhard, is about Gene-D. Perhaps the best way to conceive of the process of genetic accommodation is to recall the clustered groups of related genes associated, for example, with cell-cell recognition and adhesion. How an organism deploys its repertoire of cell-adhesion molecule variants is a problem in the regulation of a complex system. The improvement and stabilization of the new phenotype would be a complex function of many regulatory nodal points. Minute differences in both coding and non-coding regions of DNA could confer relative advantages or disadvantages in a wholly complex and context dependent fashion. Selection may be thought of as akin to that of multiple parallel processing systems that have already been entrained toward realizing that outcome that the adaptive phenotype had established, but are now competing in the ability to best achieve it. With Gene-P, and Gene-D clearly distinguished, and no longer conflated, it is all the more easy to appreciate West-Eberhard's proposal that in evolution it is the phenotype that leads the genotype.

\section{Developmental Clues and the Origins of the Human Phenotype}

Beginning with Herder and Kant in the mid-eighteenth century, there arose a 'philosophical anthropology' that located the natural basis for the higher cognitive faculties of humans not merely in a new adaptation but in a full-bodied, systematic breaking away - a loss of organismic specialization, a weakening of the senses, a weakening of the body, a loss of directed skill, a general developmental underdetermination and underdevelopment, and thereby detachment from a stable, tightly coupled, specialized relationship to particular natural surrounds. These early perceptions were given a more firm empirical foundation by the German 
philosophical anthropologists of the mid-twentieth century, such as Helmuth Plessner and Arnold Gehlen, who associated these traits with the idea that the hominid line arose by way of neotinization (or juvenalization) from our ape ancestor. German philosophical anthropology, reaching its apex between the wars, suffered a starcrossed fate, failing to succeed in gaining a foothold in the Anglophone philosophical world.

The empirical case to be made for the neoteny-based-evolution of the hominid line has only become more compelling over time and has been persuasively represented in a popular book by Clive Bromhall ${ }^{39}$ entitled The Eternal Child. The case for neoteny is put forward both by demonstrating the far greater similarity of the adult human with the very young chimp, and by considering the systematic consequences of retarding the rate of maturation at different early stages of development. With the upper body developing prior to that of the lower body, much of the morphological transition associated with the evolution of the upright posture and bipedalism can be accounted for on the basis of extending the growth phase during which legs are formed and lengthened. Similarities with the juvenile chimp but not the adult chimp include the vertical attachment of the head to the spinal column, a small jaw, more flattened face, big rounded skull, the everted lips, the sparseness and pattern of distribution of hair, the low position of nipples, the frontal position of the vulva, the retention of the outer labia and the hymen and the lack of the penis bone. It is interesting to note in this regard that bonobos, who while closely related to chimps, are distinguished from chimps by their female dominant social structure and a polymorphous sexuality that serves as the social glue of an essentially non-violent system of social relation, lie between humans and chimps on the scale of neotinization. As compared with chimps, bonobos have more delicate facial features, more rounded skulls, higher foreheads, higher voices, lighter bones, longer legs, more upright walking, a slower rate of maturation after birth, and the retention of the more frontal position of the vulva that allows bonobos, like humans, but unlike chimps, to mate face to face.

Despite stunning evidence for the role of polyphenism in general and neotinization in particular in human evolution, and despite the fact of philosophical intuitions about human detachment and underdevelopment that date back 250 years, late twentieth century thinking about the biology of human psychology, culture and cognition had been almost univocally devoted to piecemeal models of adaptation in which domain specific cognitive modules had been hypostatized very much in the image of conflationary genes taken to another level. In recent years however there has been a coalescence of recognition coming from disparate directions of the essential sociality of the human organism. Neotinization was by no means the entire basis of the evolution of the human socio-cultural capacity but it may well have been a necessary point of departure. Those who have been looking for language and reason in the brain (and the genes that code for them) have been looking for the wrong thing in the wrong place. Language, as Merlin Donald ${ }^{40}$ has taught us, didn't evolve in the 'brain box'; it evolved in socio-cultural space. What we need and are beginning to find in the human brain are the bases whereupon humans can be susceptible to each other, and thus participants and co-producers in the ever-changing games of language, with domaingeneral, cross-module 'executive' capacities for leading our own epigenetic formation, interactively, in and through the socio-cultural matrix. In the image of the 
new appreciation for the role of group selection in the evolution of sociality, neurologically based advances in social capacity could have been drawn out of the vast hominid phenotypic possibility space and stabilized in socio-cultural ritual that would be selectively advantaged in an environment of inter-group competition.

I have begun to explore elsewhere the implications of this work for philosophical anthropology and social theory. ${ }^{41}$ Suffice it for now to suggest that the shift to a new phenotype-centered biology will bring with it also opportunities, albeit not inevitabilities, for new and renewed shifts in anthropological self-understanding with all the ethical and socio-political implications that that might entail.

\footnotetext{
${ }^{1}$ Department of Sociology and Philosophy, University of Exeter, UK Lenny.Moss@exeter.ac.uk

${ }^{2}$ see N. Roll-Hansen. The Application of Complementarity to Biology: From Niels Bohr to Max Delbrück. Historical Studies in the Physical and Biological Sciences 2000; 30: 417-42; D.
}

McKaughan. The Influence of Niels Bohr on Max Delbruick: Revisiting the Hopes Inspired by 'Light and Life'. Isis 2005; 96: 507-529; A. Domondon. Bringing Physics to Bear on the Phenomenon of Life: The Divergent Positions of Bohr, Delbrück, and Schrödinger. Studies in the History and Philosophy of Biological and Biomedical Sciences 2006; 37: 433-88.

${ }^{3}$ E. Schrödinger. 1944. What is Life? Cambridge: Cambridge University Press.

${ }^{4}$ L. Moss. 2003. What Genes Can't Do. Cambridge MA: MIT Press.

${ }^{5}$ L. Moss. 2004. Human Nature, the Genetic Fallacy and the Philosophy of Anthropogesis' Jahrbuch 2004 of the Kulturwissenschafliches Institut., ed. Jorn Rusen.

${ }^{6}$ J. Maienshein. 1987. Defining Biology: Lectures from the 1890's. Cambridge MA: Harvard University Press.

${ }^{7}$ R. Falk. The Dominance of Traits in Genetic Analysis. Journal of the History of Biology 1991; 24: 457-484.

${ }^{8}$ W. Johanssen. Some Remarks About Units in Heredity. Hereditas 1923; 4:133-141.

${ }^{9}$ R. Falk. The Struggle of Genetics for Independence. Journal of the History of Biology 1995; 28:219246.

${ }^{10}$ Johanssen, op. cit. note 8

${ }^{11}$ A. Barclay. Membrane proteins with immunoglobulin-like domains - a master superfamily of interaction molecules. Seminars in Immunology 2003; 15: 215-223.

${ }^{12}$ G. Povlsen, D. Ditlevsen, V. Berezin \& E. Bock. Intracellular Signaling by the Neural Cell Adhesion Molecule. Neurochemical Research 2003; 28: 127-141.

${ }^{13}$ A. Zorn \& P. Krieg. Developmental Regulation of Alternative Splicing in the mRNA Encoding Xenopus laevis Neural Cell Adhesion Molecule (NCAM). Developmental Biology 1992; 149:197-205.

${ }^{14}$ B. Gravely, A. Kaur, D. Gunning, S. Zipursky, L. Rowen \& J. Clemens. The organization and evolution of the Dipteran and Hymenopteran Down syndrome cell adhesion molecule (Dscam) genes. RNA 2004; 10: 1499-1506.

${ }^{15}$ L. Rønn, B. Hartz \& E. Bock. The neural cell adhesion molecule (NCAM) in development and plasticity of the nervous system. Experimental Gerontology 1998; 33:853-864; L. Rønn, V. Berezin \& E. Bock. The neural cell adhesion molecule in synaptic plasticity and aging. International Journal of Developmental Neuroscience 2000; 18:193-199.

${ }^{16}$ A. Dityatev et al. Polysialylated neural cell adhesion molecule promotes remodeling and formation of hippocampal synapses. Journal of Neurosciences 2004; 24: 9372-9382.

${ }^{17}$ V. McKusick. Heritable disorders of connective tissue. III The Marfan Syndrome. Journal of Chronic Diseases 1955; 2:609-644.

${ }^{18}$ D. Milewicz, H. Dietz \& D. Miller. Treatment of Aortic Disease in Patients with Marfan Syndrome. Circulation 2005; 111:e150-e157.

${ }^{19}$ H. Dietz \& R. Pyeritz. Mutations in the Human Gene Fibrillin-1 (FBN1) in the Marfan Syndrom and related disorders. Human Molecular Genetics 1995; 4:1799-1809; P. Robinson \& M. Godfrey. The molecular genetics of Marfan syndrome and related mircrofibrillopathies' Journal of Medical Genetics 2000; 37: 9-25. 
${ }^{20}$ J. Paterson. BRCA1: a review of structure and putative functions. Disease Markers 1998; 13: 261274.

${ }^{21}$ K. Yoshidia \& Y. Miki. Role of BRCA1 and BRCA2 as regulators of DNA repair, transcription, and cell cycle in response to DNA damage' Cancer Science 2004; 95: 866-871.

${ }^{22}$ B. Billack \& A.N.A. Monteiro. BRCA1 in breast and ovarian cancer predisposition. Cancer Letters 2005; 227: 1-7.

${ }^{23}$ A-L. Barabási. 2002. Linked: The New Science of Networks. Cambridge MA: Perseus.

${ }^{24}$ H. Jeong, S. Mason \& A-L. Barabási. Lethality and centrality in protein networks. Nature 2001; 411: 41-42.

${ }^{25}$ A-L. Barabási \& Z. Oltvai. Network Biology: Understanding the Cell's Functional Organization. Nature Reviews Genetics 2004; 5: 101-114.

${ }^{26}$ S. Gilbert. 2000. Genes Classical and Genes Developmental: The Different Use of Genes in Evolutionary Syntheses' in The Concept of the Gene in Development and Evolution: Historical and Epistemological Perspectives. Beurton, Falk \& Rheinberger (Eds). Cambridge: Cambridge University Press.

${ }^{27}$ L. Moss. One, Two (Too?), Many Genes. The Quarterly Review of Biology 2003; 78: 57-67.

${ }^{28}$ L. Moss. Redundancy, Plasticity, and Detachment: the Implications of Comparative Genomics for Evolutionary Thinking' Philosophy of Science 2006; 73: 930-946.

${ }^{29}$ Ibid.

${ }^{30}$ P. Dehal et al. Human Chromosome 19 and Related Regions in Mouse: Conservation and LineageSpecific Evolution. Science 2001; 293:104-111.

${ }^{31}$ J. Baily. Recent Segmental Duplications in the Human Genome. Science 2002; 297:1003-1006.

${ }^{32}$ Barclay, op. cit. note 11 .

${ }^{33}$ Moss, op. cit. note 28; L. Moss. 2008. Detachment, Genomics and the Nature of Being Human. In New Visions of Nature: Complexity and Authenticity, Drenthen, Keulartz, Proctor (Eds), Springer International Library of Environmental, Agricultural and Food Ethics (in press).

${ }^{34}$ M. Kirschner, J. Gerhart \& T. Mitchison. Molecular "Vitalism". Cell 2000; 100:79-88.

${ }^{35}$ M. Kirschner \& J. Gerhart. 2006. The Plausibility of Life. New Haven: Yale University Press.

${ }^{36}$ G. Müller, \& S. Newman (Eds). 2003. Origination of Organismal Form. Beyond the Gene in Developmental and Evolutionary Biology. Cambridge MA: The MIT Press.

${ }^{37}$ S. Oyama, P. Griffiths \& R. Gray. (Eds) 2001. Cycles of Contingency: Developmental Systems and Evolution. Cambridge MA: The MIT Press.

${ }^{38}$ M.J. West-Eberhard. 2003. Developmental Plasticity and Evolution. Oxford: Oxford University Press.

${ }^{39}$ C. Bromhall. 2003. The Eternal Child: Has Evolution has Made Children of Us All. London: Ebury Press.

${ }^{40}$ M. Donald. 2001. A Mind So Rare: The Evolution of Human Consciousness. New York: Norton \& Company.

${ }^{41}$ L. Moss. Contra Habermas and Towards a Critical Theory of Human Nature and the Question of Genetic Enhancement. New Formations 2007; 60: 139-149.; Moss 2008, op. cit. note 33. 